\title{
Dilemmas of a rota: Google's solution
}

BMJ Quality Improvement Reports 2015; u206147.w2618 doi: 10.1136/bmjquality.u206147.w2618

\author{
Jibran S Qureshi, Fatimah Javaid
}

St Heliers and Epsom Hospital NHS Trust, London, United Kingdom

\begin{abstract}
Doctors take many forms of leave to further career training. Many hospital rotas omit these and are not updated frequently to reflect swaps in on-call duties. As a result, confusion arises with reduced staffing levels leading to sub-optimal patient care. Using Google Sheets, rota's can be modified to be team specific, remotely accessed, and edited by any device with an internet connection and web browser. A qualitative pre and post-implementation questionnaire assessed the usefulness of the online rota.
\end{abstract}

The rota was trialled on a surgical and medical team containing foundation year, core medical, core surgical, and registrar trainees. The respective surgical and medical rotas were copied, modified and pasted into a Google Sheet to contain only team specific information including all forms of leave. A link was emailed allowing teams to access and edit the rota. Post-implementation questionnaire $90 \%(n=10)$ stated they could access it remotely and $100 \%$ felt the new rota was very easy to use. Finally, $80 \%$ stated the Google Doc rota was a definite improvement on the old trust rota.

The rota was then trialled on an ITU SHO rota $(n=8)$. The results were reproducible with $88 \%$ feeling it addressed their requirements and stating it was an improvement on their old rota. As a result of the implementation appropriate staffing levels were maintained on the ward. The key advantages of the Google rota are that it included all forms of leave and helped ensure adequate staffing levels on the ward. The remote nature means easy access even if off-site and can be viewed easier on smart phones. The different privacy settings ensure security of document. The rota is updated in real-time giving an accurate representation of staffing levels - vital for service managers. The software is free to use, allowing it to work within current hospital IT framework.

\section{Problem}

As a foundation trainee, I found that the medical and surgical rota was often a cause of confusion and frustration. Due to the European Working Time Directive (ETWD), doctors are often subjected to rotas whereby each week the working hours are different and as a result have compensatory zero days to comply within regulations. This leads to a complex and difficult to follow rota. At St Heliers Hospital, a district general in South West London, both medical and surgical rota's were saved centrally on the hospital network and could only be viewed while on a trust computer. The trust rota included the shifts of every junior doctor who made up the surgical or medical department. This led to a very large spreadsheet, which was complicated to follow even on a large computer screen.

This sheet was often not updated to reflect swaps in on-call duties and the many forms of leave taken. This often led to confusion and sub-optimal staffing levels as leave or on-call swaps had been approved but not updated on the rota and teams were unaware of this. We currently organise our rota in one of four ways:

1. Inform colleagues verbally/ face-to-face. This is not noted anywhere leading to teams being unaware those colleagues are away

2. Inform colleagues via email. Email's are then lost or unread leading to the above

3. Team specific rota is made using Excel but only accessible via a trust PC. This reduces the above errors, however if not on-site then it cannot be viewed

4. An excel spreadsheet is emailed to team members. This leads to multiple copies of the rota being sent round, often confusing which is the most up-to-date. The medical trust rota was emailed out at the end of every week to show who was on-call for the coming weekend. This often led to nasty surprises as swaps, which had been done were not reflected on the rota. As colleagues did not always check the email, it sometimes led to the on-call team being a team member short, leading to increased work load and pressure on the on-call team.

\section{Background}

The 1993 European Working Time Directive (EWTD) was created in order to improve doctor and patient safety by establishing a 48-hour working week with rest periods and holiday allowance. At first implementation in the UK in 1998 it did not include junior doctors but was later enforced in August 2009 reducing working hours per week from 56 to 48 hours.[1] Shorter shifts, rest days and regular breaks result in less doctor exhaustion, better clinical precision and efficient working practice.[2] 33\% fewer medical errors occur on EWTD compliant rotas as compared to the traditional 56 hour/week system in addition to fewer non-intercepted potential adverse events.[3] Emphasis on the importance of personal development including study leave, simulation training and teaching courses is necessary to improve quality and safety in healthcare. These training days are often not updated or omitted on hospital rotas and the onus is placed upon the doctors themselves to liase with 
BMJ Quality Improvement Reports

administrative and clinical staff in order to take educational leave. This can often lead to confusion at late notice, particularly with respect to on-call duties. In essence, the consequent reduced staffing levels lead to sub-optimal patient care. Modern technology has previously been utilised to design doctor rosters in agreement with the EWTD. Software created in Oxford was used to implement the 48 hour working week into an senior house officer (SHO) rota in accordance with annual leave requests. Although useful in generating a rota with specified leave, it is complicated to use if unfamiliar with computer programming.[4]

\section{Baseline measurement}

A 13 point questionnaire was sent to a surgical and medical team asking them to identify the current strengths and weaknesses in their current respective rotas. It also determined if they had created their own team specific rota already and the specific features it included. It was distributed to registrars, core trainees and foundation year doctors. A total of nine respondents were identified.

The results showed that the current trust surgical rota failed in certain aspects. $89 \%$ of respondents reported the rota did not state which of their colleagues was on annual leave and the length of time they would be away. One hundred percent stated that the rota did not tell them which site an SpR or SHO were on in addition to whether they were in clinic or in operating theatre, and $100 \%$ reported the rota did not tell them if a colleague was on a simulation, training, or intermediate life support course day.

Forty-four percent $(n=4)$ of respondents had created their own team specific rota. Of this only $50 \%$ of them covered all the information needed on the rota. $50 \%$ of these rotas could be updated on a trust computer, with the remaining $50 \%$ via email. None of the team specific rotas could be updated directly online.

See supplementary file: ds4323.xls - "Pre-implementation questionnaire results"

\section{Design}

It became clear that this was a significant issue for many teams. It was not unfamiliar to overhear another surgical or medical team confused over why the team was understaffed on a particular day and then later realising a colleague had taken leave. The rota administrator had approved the leave but the team were unaware of it.

I decided that a team specific rota would be ideal for my surgical and medical teams. I was aware that Google had created Google Sheets, a basic version of Microsoft (MS) Excel. Its advantages include that it can be remotely edited, shared, and updated in real time. The spreadsheet is saved centrally and automatically on Google servers. Furthermore, it can be viewed on any device which has a web browser and Internet connection, ie PC, laptop, tablet, and smartphone. This is something an MS Excel spreadsheet is not able to do.
I created an online spreadsheet in the following steps:

- You need a Gmail account to access Google Sheets, which I already had

- I used our existing surgical/medical rota and copied it into Google Sheets

- I then removed the columns that were not relevant to our team, such as other teams rotas

- I added in an extra column for leave, whether it is study leave, annual leave, taster weeks etc

- I then sent a link to the SpRs, core trainees (CT), and foundation year doctors (FY) in the teams

- The link was initially set as being able to view and edit the rota by anyone who was given the link. The advantage here was that no person needed to "login" to view the rota or have a Gmail account.

Now the rota was online and functional. The next step was sitting down together as a team, showing them how the rota worked and how it could be edited. The whole team then added their different forms of leave, such as study leave, training days, conferences etc. Specialist registrars also included when they were around to do ward rounds. Both medical and surgical teams were fully committed with this idea and individually contributed to the Google Sheet.

Within three weeks, $90 \%$ of leave had been booked and I had created a fully functional online rota which was remotely accessible and editable. The rota was then trialled on an intensive care unit rota for SHO's. Similar steps were taken as above with a run through given of how the rota works.

These rotas are still in use even after I have left the respective teams, and I have introduced this rota to other teams I have been in since. In order for it to be sustainable, it requires one person in the team to be trained in making the Google Sheet. This does not take more than 10 minutes. It also requires the team to be fully engaged with the rota and make amendments to the rota as leave is booked.

The long-term plan is for all rotas to become online. This would allow easy accessibility of the rota whether at home or work. Teams should also have their own team specific rotas that cater to their team's specific characteristics. If this is shared with rota coordinators then they will have an accurate reflection of staffing levels in the hospital.

\section{Strategy}

PDSA cycle 1: The first cycle, as stated above, was introduced immediately to the medical and surgical teams. The results were very encouraging, with users finding it very easy to use and access. Feedback noted one user stated they could only access it via email. A recommendation was that users would like to be notified each time a change is made to the rota. 
PDSA cycle 2: A post-measurement was carried out at this point. The user who said they could only access the rota via email was walked through again how to use the rota and also showed how to make a bookmark link on their web browser so they could access it quickly in the future. Settings were amended so that each time the spreadsheet was modified an email was sent to all the users notifying them.

PDSA cycle 3: The rota was then trialled on an ITU SHO rota. Notifications were sent automatically to all participants when changes were made to it. Participants also bookmarked the email link allowing them to access it quickly. There was no negative feedback regarding the rota. A post-measurement was carried out four months after implementation.

\section{Results}

A post-implementation 13 point questionnaire was sent to 10 team members of both the medical and surgical team, who again ranged from SpRs, CTs, and FYs. When asked what was important for their team specific rota to provide, the responses included:

- Awareness of staff who are in clinic/surgery and at what times

- On-call duties

- Annual leave

- Helping establish good continuity of care

- An up to date rota

- Awareness and knowledge of which seniors were available to access on the ward.

Seventy percent stated the Google Sheet addressed all of their rota requirements. Seventy percent said it was excellent at identifying who is on annual leave and their length of time away, with a further $30 \%$ saying it was adequate. None of the respondents felt the rota had insufficient detail or worst.

Sixty percent felt it was excellent at identifying which $\mathrm{SpR}$ or $\mathrm{SHO}$ was on-site, $20 \%$ felt it was adequate, and $20 \%$ felt it had insufficient detail. A likely reason for this was the fact not all the SpR's for the surgical team inputted their dates in the rota. This understandably led to some confusion regarding who was on-site at a given time in the rota. Seventy percent stated the rota was excellent at informing staff if a colleague was on a simulation training/study day/taster week or intermediate life support course with a further $20 \%$ stating it was adequate.

Encouragingly, $90 \%$ were able to access the rota remotely via an internet connection and $100 \%$ were able to update the rota remotely. Only $50 \%$ stated that for most of the time, if not all the time, members of the team were informed when the rota was updated. This is due to notifications on the Google Sheet not being made active before that point. After receiving this feedback, users were educated on how to activate the notifications. This was then implemented post feedback and this issue was resolved.

One hundred percent stated the new rota was very easy to use and $80 \%$ felt it helped ensure adequate junior cover at all three sites most of the time. Promisingly, $80 \%$ felt the new Google Sheet rota was a definite improvement on the old trust rota.

The rota was then trialled on an eight person ITU SHO rota $(n=8)$. The feedback from this was very encouraging. Eighty eight percent stated the Google Sheet addressed all of their rota requirements. Seventy five percent said it was excellent at identifying who is on annual leave and their length of time away, who is on site, and who is on study leave. Once again $100 \%$ stated the new rota was very easy to use and $80 \%$ felt it helped ensure adequate junior cover at all three sites most of the time. Eighty eight percent agreed the Google Sheet rota was a definite improvement over the old trust rota.

See supplementary file: ds4363.xlsx - "Google rota postimplementation feedback"

\section{Lessons and limitations}

The success of the Google rota was due to the engagement of the core trainees, foundation years and SpR's and their investment in its development and maintenance. They understood first-hand the importance of adequate staffing levels on the wards and how this impacted on patient care. Furthermore, they knew it impacted on continuity of care. By giving a short introduction and walk through of the rota, the teams quickly became familiar with its utilisation. It allowed for early identification of when leave could be taken in addition to potential under staffing on a given day.

You only get out what you put in. If all team members do not contribute to the rota, then evidently it is not updated accordingly. This consequently results in a misrepresentation of staffing levels. This was the pitfall in regards to the surgical rota, as some SpRs did not input the data regarding when they were on-site to do ward rounds. On the ITU rota this occurred due to colleagues not always inputting their annual/study leave on the rota.

The intervention is cost effective on many fronts. No new software is required to use Google Sheets, just an up to date browser. Furthermore, there is now a dedicated Google Sheet app available for Android and Apple iOS phones allowing easy access and viewing on a smartphone. It takes some of the responsibility away from the rota co-ordinator, allowing doctors to identify early any short staffing incidences in the rota. By helping ensure adequate staffing levels on the ward, it reduces the incidence of having to hire a locum at short notice, which is very expensive to the trust. If advanced notice is given regarding short staffing on the ward and a locum is needed, then it gives the rota coordinator time to find an appropriately trained locum. This may be either within the trust bank staff or externally but importantly early planning will help to maintain good quality patient care.

\section{Conclusion}


Using Google Sheets to create a team rota enables it to be team specific, allowing it to be smaller, concise, and easier to view than previous practice. Current rotas can easily be transferred to the application and there is no need for new software to be installed or purchased. There is no difficulty in accessing the rota as it can be viewed by current web browsers used in most hospital trusts. There is no need to constantly edit and re-save the data, as cloudcomputing centrally saves any updated information.

The linking with a dedicated mobile app further increases its accessibility and usability and allows staff to check the rota remotely and off-site. Anecdotally it has been noted to raise moral within teams as they feel well supported and informed. The feedback clearly shows it is easy to use and access whilst being an improvement on the previous trust rota.

Ultimately, the use of this simple technology will help bring rotas back in to hands of doctors and empower them to take ownership of the service they provide whilst complying with EWTD.

\section{References}

1. Bma.org.uk. BMA - European Working Time Directive [Internet]. 2014 [cited 20 November 2014]. Available from: http://bma.org.uk/practical-support-at-work/ewtd

2. Royal College of Physicians. Designing safer rotas for junior doctors in the 48-hour week. Prepared on behalf of a multidisciplinary working group by Horrocks $\mathrm{N}$ and Pounder R. London: Royal College of Physicians, 2006.

3. Cappuccio F, Bakewell A, Taggart F, Ward G, Ji C, Sullivan $\mathrm{J}$ et al. Implementing a $48 \mathrm{~h}$ EWTD-compliant rota for junior doctors in the UK does not compromise patients' safety: assessor-blind pilot comparison. QJM 2009;102(4):271-82.

4. Todd B. A computer program for generating New Deal compliant SHO rosters. Emerg Med J 2004;21(4):487-90.

\section{Declaration of interests}

Nothing to declare.

\section{Acknowledgements}

Dr Pamela Leventis for her unwavering support and encouragement. 